\title{
A FUZZY CONGESTION CONTROLLER TO DETECT AND BALANCE CONGESTION IN WSN
}

\author{
Ali Dorri ${ }^{1}$ and Seyed Reza Kamel ${ }^{2}$ \\ ${ }^{1}$ Department of Computer Engineering, Mashhad Branch, Islamic Azad University, \\ Mashhad, Iran \\ ${ }^{2}$ Department of Computer Engineering, Mashhad Branch, Islamic Azad University, \\ Mashhad, Iran
}

\begin{abstract}
A Wireless Sensor Network (WSN) is collection of wireless sensors with limited memory, processing and energy supply. Based on application, sensors distribute in a wide geographically area in order to collect information and transmit the collected data packets toward a base station also called Sink. Due to the relatively high node density and source-to-sink communication pattern, congestion is a critical issue in WSN. Congestion not only causes packet loss, but also leads to excessive energy consumption as well as delay. To address this problem, in this paper we propose a new fuzzy logic based mechanism to detect and control congestion in each grid in WSN. In the proposed approach, sink select one node in each grid as Monitor Node. In addition, sink defines congestion candidate grids. Each Monitor Node in congestion candidate grids continually monitors the network and fetches the fuzzy controller inputs in order to determine level of congestion in each grid. Based on the congestion level, packets forward through the grid or relay nodes. Simulation results show that our approach has higher packet delivery ratio and lower packet loss than existing approaches.
\end{abstract}

\section{KEYWORDS}

Fuzzy Logic, Wireless Sensor Network, Congestion Control, Packet Lost.

\section{INTRODUCTION}

A Wireless Sensor Network (WSN) consists of spatially distributed autonomous wireless sensor nodes to cooperatively monitor physical or environmental conditions, such as temperature, sound and pressure. In addition, WSN is a network made of hundreds or thousands of sensor nodes which are densely deployed in hazardous/unattended environment with capability of sensing, computing and sending information wirelessly to the base station (also called sink) via neighbour nodes. Figure 1 shows a WSN that collect information and send it to the sink. 


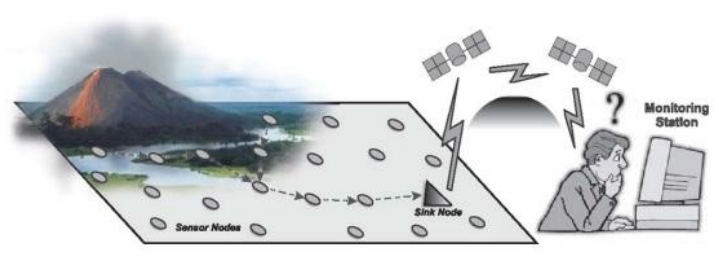

Figure 1. Wireless Sensor Network [1]

Each sensor has four different units which are: processing unit, memory unit, communication unit and power unit. Figure 2 presents the four important units in a sensor. All sensor units uses power supply. All received packets process in processing unit and some information save in memory unit. In WSN special applications, once an event occurs, a sudden surge of data traffic will be triggered by all sensor nodes in the event area, which may easily lead to congestion.

Congestion in WSN has a negative impact on network performance. It increases the packet loss, end-to-end delay and wastes nodes energy. When a packet is lost, source node must retransmit it again. Therefore, node's energy is wasted and network lifetime will be decreased $[2,3]$.

However, some characteristics of WSN, such as constrained resources and leak of centralized coordination, make the congestion problem in WSN more challengeable than any other networks. In WSN all nodes send their sensed data to the sink. This flow of packet (also called source-to-sink traffic), increase congestion probability and energy consumption in nodes near the sink. The reason is that, the neighbouring nodes of the sink should forward other nodes packets in addition to their own traffic [4,5].

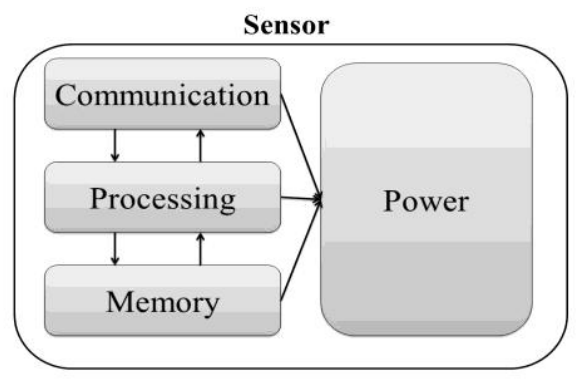

Figure 2. Sensor's Different Units

To address these challenges, we present a fuzzy congestion control. In the proposed approach, the network is divided into grids by the sink. Then, the sink specifies congestion candidate areas by use of a calculated threshold. In each congestion candidate area, a Monitor Node (MN) uses a fuzzy controller to detect and avoid congestion in its grid. Congestion level of each grid maybe different from other grids and if the congestion level of any congestion candidate area reaches to the acting level, the Border Node forwards packets out of the congested area. Therefore, congestion and packet loss will be decreased. Simulation results show that the presented fuzzy-based system decreases the packet loss in congested area and increases packet delivery ratio to the sink. The remainder of this paper is organized as follows: section 2 presents a literature review of the congestion detection and control mechanisms. Section 3 provides detailed description of the proposed fuzzy controller approach. Performance evaluation of the 
proposed approach is presented in section 4 . Finally section 5 concludes and discusses the future directions of this research.

\section{RELATED WORKS}

In literature many congestion control schemes have been proposed for WSN. Congestion control schemes for WSN either focus on MAC layer or on both MAC and network layer [6]. Authors in [7] presented an approach based on a threshold. This threshold refers to ratio of received packets to serviced packets. In this approach each node has a priority. To detect congestion, both threshold and priority of nodes are influenced. Proposed approach is efficient in term of Quality of Service (QOS) as it sends data through multipath. Authors in [8] presented a Medium Access Control (MAC) technique to coordinate the access of nodes to the shared medium. It uses the queue buffer length of the sensor nodes to estimate the congestion. Then the traffic dynamically disseminates along with classifying nodes into different priority classes to provide a congestion-free routing path to the destination with improved QOS. In addition, it uses multiple forwarder traffic diffusion, which has advantages like increasing network reliability and reducing congestion. In [9] an optimal routing algorithm that allows optimizing transmission between the peripheral nodes and central node is presented, in order to increase the residual energy of the network. This protocol only aims to provide routing fidelity and does not address time transmission requirements. Authors in [10] presented a cross-layer congestion controller that has three parts: 1) multipath routing 2) adjusted ratio 3) application oriented design. In this algorithm, each node has multiple downstream nodes to be transmitted. The probability of forwarding nodes and the rate of sending packets can be dynamically adjusted according to the congestion state. Authors in [11] presented a novel approach based on bird's behaviour. The proposed approach is simple to implement at the individual node, involving minimal information exchange. In addition, it displays global self-properties and emergent behaviour, achieved collectively without explicitly programming these properties into individual packets. Performance evaluations show the effectiveness of the proposed Flock-based Congestion Control (Flock-CC) mechanism in dynamically balancing the offered load by effectively exploiting available network resources and moving packets to the sink. Furthermore, Flock-CC provides graceful performance degradation in terms of packet delivery ratio, packet loss, delay and energy consumption under low, high and extreme traffic loads. In addition, the proposed approach achieves robustness against failure and also has scalability in different network sizes and outperforms typical conventional approaches. Authors in [12] present a fuzzy based congestion controller. The presented approach which is called Fuzzy Based Adaptive Congestion Control (FBACC) uses buffer occupancy, participants and traffic rate as the inputs of fuzzy system. Using these three parameters fuzzy system estimates the congestion ratio and drops some of packets to balance the traffic. Compared to other approaches, this approach has more preciously. In addition, presented approach saves packet transmission energy by minimizing packet loss. Authors in [13] proposed a fuzzy logic congestion controller which detects congestion using buffer occupancy and congestion index. In this approach, fuzzy system periodically calculates the congestion degree and uses it to adjust upstream traffic rate.

Figure 3 presents a summarization of discussed approaches for congestion control in WSN. 


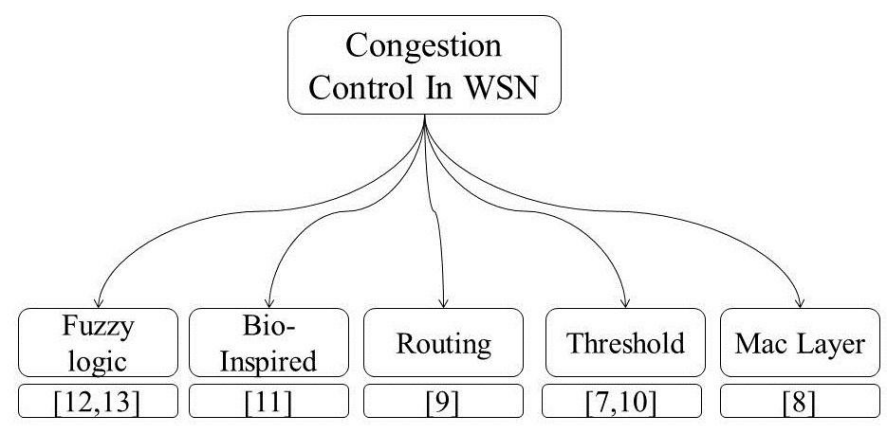

Figure 3. Congestion Controller mechanisms in WSN

\section{The Proposed APPROACH}

In previous section, we presented a literature review on congestion controller mechanisms in WSN. In this section, we briefly discuss our fuzzy-based congestion controller. The basic idea of a fuzzy controller is presented in Figure 4. Fuzzy controller can increase the accuracy in congestion detection as it uses different metrics and membership functions in order to detect congestion. Each fuzzy controller convert received crisp inputs into fuzzy inputs using a Fuzzifier. Then, by using a rule base fuzzy system determines congestion level. Rules consist of several rules that are formed by use of input metric's membership functions. The output of fuzzy system converts to crisp input using a Defuzzifier.

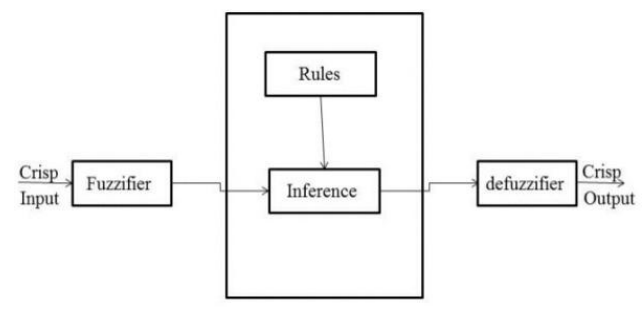

Figure 4. Fuzzy Controller System Functionality

In presented approach, fuzzy logic controller is considered as the kernel of the algorithm. It is associated with the Monitor Nodes (MNs) to detect congestion level, based on input information which comes from the network. In the propose approach, $\mathrm{MN}$ continuously monitors its grid and fetches fuzzy controller's input metrics. Therefore, the congestion level of each grid is calculated dynamically by using different metrics. We describe our fuzzy-based congestion controller in three phases that are as follows:

1) Congestion candidate generation

2) Congestion identification

3) Generating new phase

We discuss each phase briefly in the rest of this section. 


\subsection{Congestion Candidate Generation}

After the sensors establishment, based on geographically position of the sensors in network, sink divide network into some equal grids. In lots of WSN applications sensors randomly distribute in the area in order to monitor the network. Therefore, the number of sensors in each grid will be different from other grids. After dividing the network into grids, sink allocates an ID to each grid called Grid_ID and sends it for each grid members. Sink cheese a sensor in each grid as the Monitor Node (MN). Responsibility of the MN is to monitor its own grid continuously and fetch fuzzy system's input metrics. Sink choose the sensor with highest reminded energy as the MN. In the case that two sensors had the same reminded energy, the nearest sensor to the sink is chosen as the MN. The reason of choosing highest energy reminded sensor as the $\mathrm{MN}$ is that the MN monitors its grid continuously that consumes the MN's energy. Therefore, the MN consumes more energy than the other sensors.

The presented fuzzy system uses each grid's density in order to detect congestion candidate areas. Therefore, the first duty of each MN is to calculate its grids density and send it to the sink. Whoever, at the beginning, sink knows the number of sensors in each grid, but in the case that a sensor dies as lack of energy, the $\mathrm{MN}$ recalculate its grids density and send it for the sink. When each sensor's energy reaches to the alarm level, it sends a packet for MN and aware MN of its death. The alarm level's energy is enough just for sending a packet to MN.

In order to calculate grids density, the MN puts Grid_ID in a packet and broadcast it for its neighbours. Each sensor that receives the packet compares its own Grid_ID with the Grid_ID of the received packet. If both are the same, the sensor sends a replay to the $\mathrm{MN}$ and then rebroadcasts packet. Sensor drops the packet, either if it received the same packet before or if packet's Grid_ID is not the same as node's Grid_ID.

After receiving all grids densities, sink calculates grid's density average and uses it as a threshold for defining congestion candidate grids. Each grid with higher density than the calculated threshold is marked as the congested candidate area. If any change in number of sensors happened, sink recalculates a new threshold and updates congested candidate grids. High density regions have higher congestion probability and higher collision and packet lost rate. A summary of this phase is shown in Figure 5.

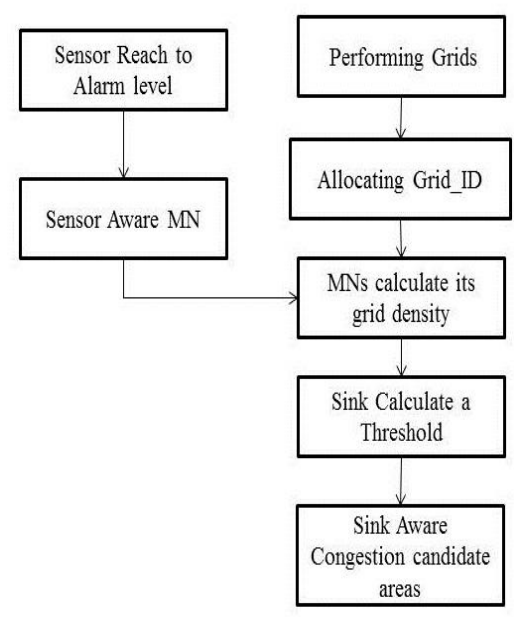

Figure 5. Steps for Congested Region discovery 


\subsection{Congestion Identification}

In each MN there is a fuzzy-based system, which controls the congestion in grid and balance traffic in order to reduce the congestion. Fuzzy system uses three different metrics as inputs to determine congestion level. Fuzzy controller for each MN is shown in Figure 6. Based on the congestion level, packets forward either through the grid or through the relay nodes.

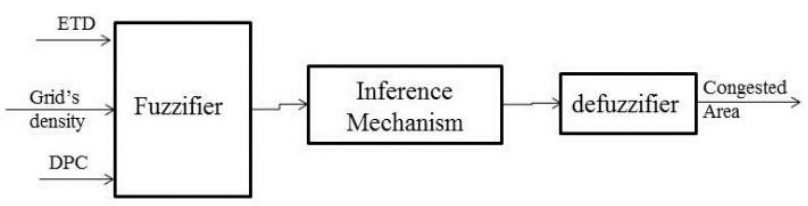

Figure 6. The Proposed Fuzzy Logic Controller

Three fuzzy input parameters are as follows:

ETD (Extended Transmission Delay): The ETD metric is the transition time that is required to transmit a packet to the next hop. The metric is calculated using the convex combination presented in Formula.1.

$$
E T D(t)=\alpha * \operatorname{Delay}(t)+(1-\alpha) * E T D(t-1) \quad \text { Formula. } 1
$$

In this formula, Delay( $t$ ) determines delay in time $t$, and $\alpha$ is weight value. $\alpha$ is used to determine the priority of delay or previous ETD.

Grid's Density: High density of sensors in each grid can easily increase congestion probability. In addition, grids density may increase or decrease during network lifetime. The reason is that a sensor may die or new sensors may add to the network. Number of sensors in each grid has a direct effect on congestion. Therefore, number of sensors is an important metric in the proposed fuzzy system.

DPC (Dropped Packets): Dropped packets, refers to the number of lost packets in each grid. Packet loss is one the results of congestion. Therefore, increasing packet loss means increasing congestion.

These parameters have membership functions that are presented in Figure 7. By use of these membership functions, a rule base is designed for fuzzy system. When MN calculates inputs, fuzzy system applies them in the rule base. Fuzzy system output determines three different actions that are as follows:

Action1) relay all packets through the grid

Action2) relay half of the packets through the grid and half through the relay nodes

Action3) relay all packets through the relay nodes

We will discuss the relay nodes and these actions in the next phase. Using presented approach in this phase; fuzzy controller detects congestion and tries to reduce the packets in the congested grid. 

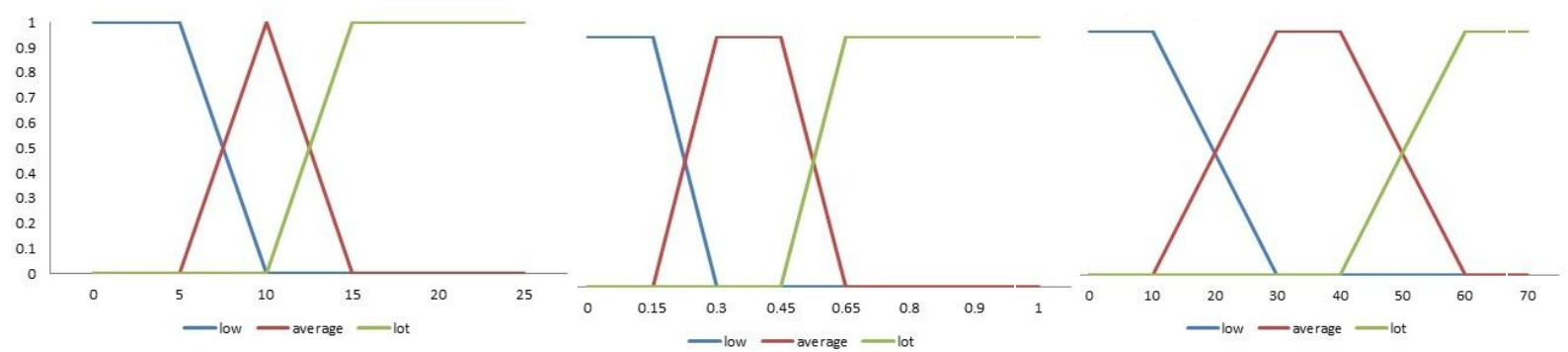

a) Grids Density

b) ETD

c) Dropped packets

Figure 7. Membership Functions Diagrams

\subsection{Generating New Path}

When fuzzy system determines the action, the MN performs the specified action. If there is no congestion, output will be action1. In this case, there is no need for the MN to perform any action. But for other two levels, the MN must informs its grid's Border Node (BN) about the congestion level. At the first time, the MN sends a packet to the sink and asks for its BN's ID. The sink selects the last node in grid as the BN. The detail of selecting BN is presented in [15]. Figure 8 shows the position of BNs and MNs in the network. After selecting the BN, the sink informs the $\mathrm{MN}$ of its grids $\mathrm{BN}$. BN has the responsibility of relaying the packets either through the grid or through the relay nodes. The BN relay packets based on action level and this will continue until congestion level in the grid reduce to action1. When the $\mathrm{BN}$ gets informed of any changes in congestion level by the $\mathrm{MN}$, it sends packets through relay path based of congestion level. Since radio frequency of relay nodes is different from ordinal sensors, sending packet using relay path has no effect on congestion in the grid. Sink selects the best relay path for BN based on [15].

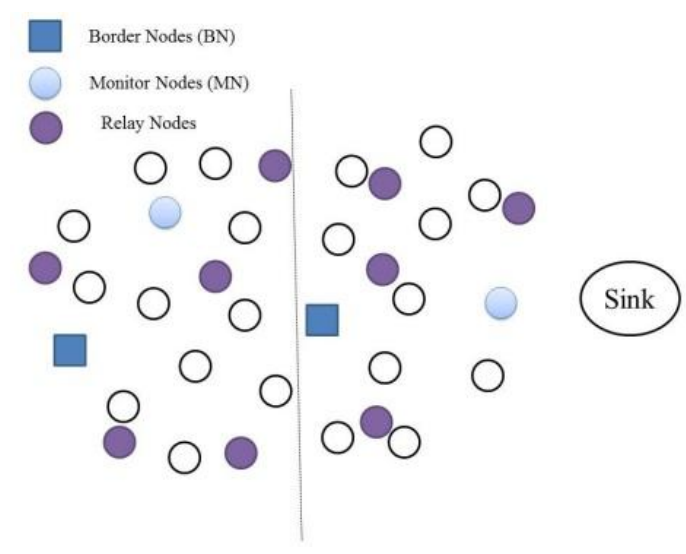

Figure 8. Example of BN and MN Assignments

In this section, we briefly discussed our fuzzy based congestion controller approach. A summary of the proposed approach is shown in Figure 9. 


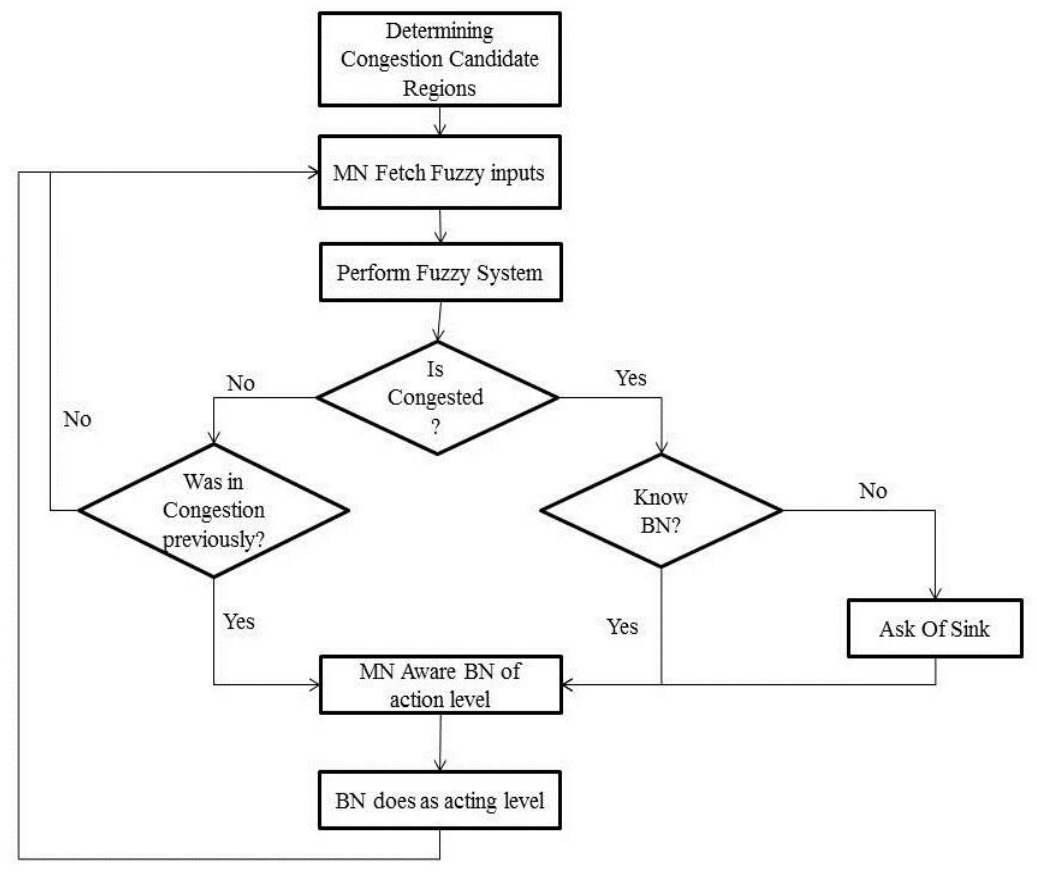

Figure 9. The Proposed Approach

\section{SIMULATION RESULTS}

To show the advantages of the proposed approach in compare with the Base Work (BW) [14], both approaches implemented using Opnet Modular 14.5 simulator. The simulation was performed using a WSN of size $300 \mathrm{~m} * 300 \mathrm{~m}$. Table 1, lists simulation parameters used in our study.

Both the BW and our approach were implemented using two different scenarios. For both scenarios two parameters have been measured and evaluated. These parameters are as follows:

Packet delivery: number of packets reached to the sink

Number of dropped packets: number of packet lost because of congestion in congestion candidate grids.

Retransmitted packets: When a packet gets lost, the source node must retransmit it again until the packet reaches to the destination. Packet retransmission wastes sensors energy as the sensors have to send packet for second time and this can easily decrease sensor's energy. In addition, sending retransmitted packets increases congestion and number of packet loss in the network. 
Table 1. Simulation Parameters.

\begin{tabular}{ll}
\hline Parameter & Value \\
\hline Simulation duration & $180 \mathrm{sec}$ \\
Number of nodes & 26 \\
Transmission range & $20 \mathrm{~m}$ \\
Traffic type & CBR(UDP) \\
Packet rate & 2 packets/sec \\
Data payload & 512 byte/packet \\
Number of relay nodes & 6 \\
\hline
\end{tabular}

These parameters are highly important in congestion control approaches as one of the aims of congestion approaches is to reduce number of lost packets and decrease retransmitted packets in WSN. In WSN, communication unit consumes more energy than the other units. It means transmission is the costliest work in sensors in the case of energy consumption. Therefore, decreasing extra transmission packets can increase network lifetime.

Figure 10 presents packet delivery rate for our approach and our base work.

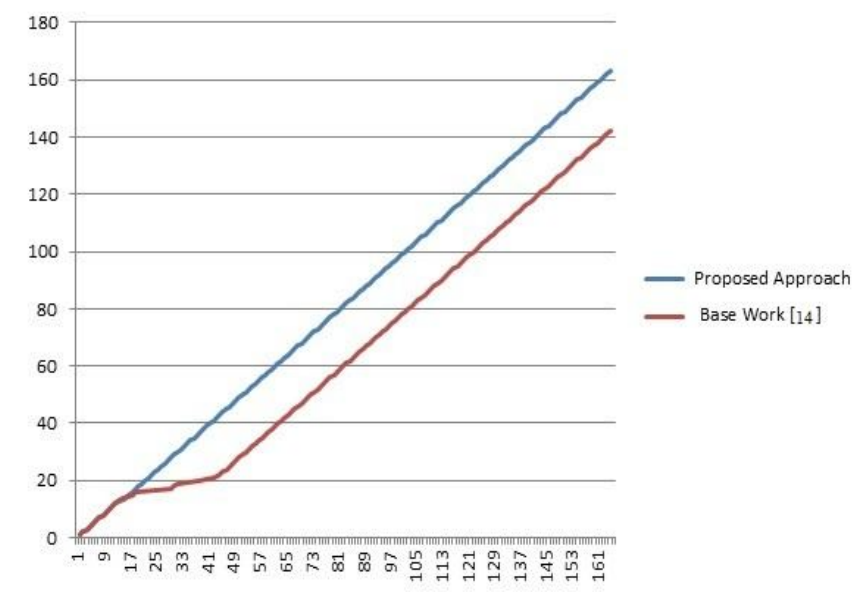

Figure 10. Packet Delivery Rate

In this figure horizontal axis refers to time and the vertical axis, to number of packets delivered to the sink. In the first seconds of simulation, both approaches have the same packet delivery ratio to the sink. Since fuzzy system uses a rule base and three input parameters to detect congestion level, any changes in one or more than one of these metrics can specify a new rule in rule base and even change the congestion level. Therefore, the fuzzy controller determines a level of congestion and forwards some packets through relay nodes. However, the base work hasn't detected any congestion yet. After a while, base work detects congestion and forwards all packets through the relay nodes. In this time, fuzzy system detects a higher congestion level and like base works forward all packets through the relay nodes. Therefore, presented approach has higher packet delivery rate.

In order to compare packet lost rate, we uses the packet lost ratio in each congested grid in both approaches as presented in Figure 11. 


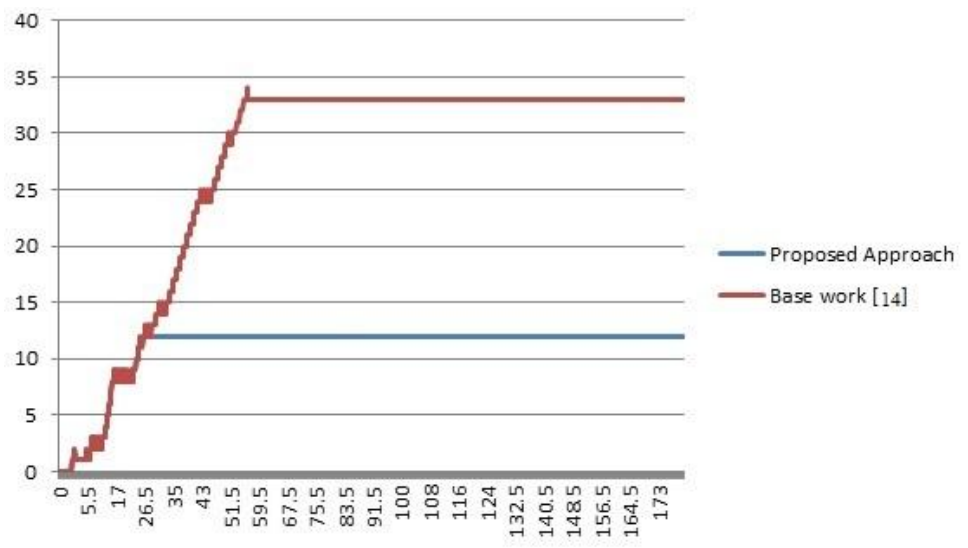

Figure 11. Packet Loss in Congested Grid rate

Vertical axis refers to packet lost rate in congested grid, and horizontal axis refers to time. In the first seconds of simulation number of lost packets is the same in both approaches. By increasing number of lost packets the fuzzy system detects a level of congestion and balance the traffic in the congested grid by forwarding half of the received packets through relay nodes. As relay nodes have different frequency from ordinal sensors, congestion in grids has no effect in relay path packets. Therefore, there will be no packet lost in relay path. The dentate mode of the diagram comes from difference in number of packet lost in the grid.

The simulation results for number of retransmitted packets in the source sensor for both approach is presented in Figure 12.

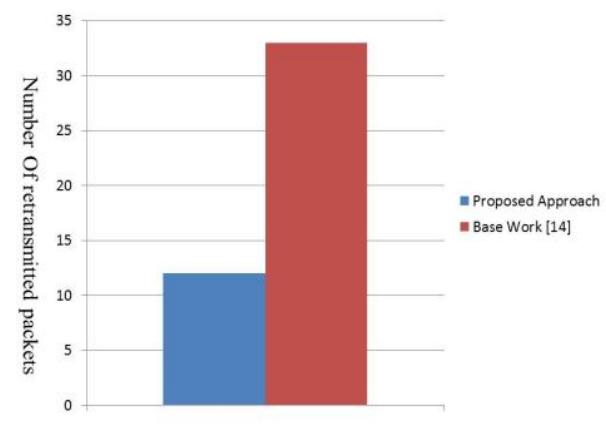

Figure 12. Number of retransmitted packets in the source

Referring to Figure 12 the number of retransmitted packets in the source node in fuzzy-based approach is lower than base work. This amount of packets gets lost in the first seconds on the simulation and changed the congestion level in fuzzy system. As we mentioned before packet retransmission consumes more energy, wastes network bandwidth and increases congestion and collision probability. Therefore, retransmitted packets increase congestion probability. Furthermore, retransmission consumes more energy of the sensors and decreases network life time.

Referring to presented simulation results, it's understandable that the presented fuzzy system approach can detect congestion in an acceptable time and sooner than the base work [14]. In 
addition, it can balance the traffic in a grid by forwarding half of packets through relay path. By decreasing the number of retransmitted packets, it increases network lifetime. The presented fuzzy controller is flexible and can adopt for any network. Based on application, membership function of each fuzzy input metric can updated, then updated membership functions form new rule base for the network. In addition, accuracy of fuzzy system can increase by defining new membership functions with more states. Our membership functions have three states therefore, our rule base contains nine different rules. Increasing the states of membership functions, increased the number of rules in rule base and in addition, increases the number of congestion levels output. Therefore, new actions and congestion levels can defined.

\section{CONCLUSION AND FUTURE WORK}

Wireless Sensor Network (WSN) is a set of sensors that distributed in an area in order to monitor the environment, In WSN, sensors send their packets through the neighbour nodes to a base station also called Sink. Therefore, the traffic nature in this network is from sources to the sink that easily increased congestion, packet lost and energy consumption in network especially in sensors nearer to the sink. Congestion has negative impacts in WSN as it increases packet lost probability and energy consumption in the sensors. In this paper, we proposed a novel approach based on fuzzy logic to determine and balance congestion in grid-based WSN. The fuzzy controller performs in each grid's MN and uses three metrics to determine congestion level of the grid. Referred to congestion level packet forwards through the grid or the relay path. Since the frequency of the relay nodes is different from ordinal sensors, congestion has no effect on them. Simulation results show that presented approach can balance and detect congestion with better performance in compare with our base work. Since energy is the most important parameter in WSN, as our future work, we decided to decrease energy consumption of our work and increase network lifetime.

\section{REFERENCES}

[1] L.A. Villas, A. Boukerche, H.S. Ramos, H.A.B.F. de Oliveira, "DRINA: A Lightweight and Reliable Routing Approach for In-Network Aggregation in Wireless Sensor Networks", IEEE Transactions on Computers, (Volume:62, Issue: 4 ), 2013.

[2] R. Annie Uthra, S.V. Kasmir Raja, " Energy Efficient Congestion Control in Wireless Sensor Network", Recent Advances in Intelligent Informatics Advances in Intelligent Systems and Computing Volume 235, pp 331-341, 2014.

[3] Sh. Borasia, V. Raisinghani," A Review of Congestion Control Mechanisms for Wireless Sensor Networks", Technology Systems and Management Communications in Computer and Information Science Volume 145, pp 201-206, 2011.

[4] C. Karakus, A.C. Gurbuz, B. Tavli," Analysis of Energy Efficiency of Compressive Sensing in Wireless Sensor Networks", Sensors Journal, IEEE (Volume:13, Issue: 5 ), 2013.

[5] C. Sergiou, V. Vassiliou, "Study of lifetime extension in wireless sensor networks through congestion control algorithms", IEEE Symposium on Computers and Communications (ISCC), 2011.

[6] J. Zhao, L. Wang, S. Li, X. Liu, " A Survey of Congestion Control Mechanisms in Wireless Sensor Networks", in Sixth International Conference on Intelligent Information Hiding and Multimedia Signal Processing (IIH-MSP), 2010.

[7] C. Wang, B. Li, K. Sohraby, M. Daneshmand, "Upstream congestion control in wireless sensor networks through cross-layer optimization". IEEE Journal on Selected Areas in Communications, (Volume:25, Issue: 4 ).

[8] U.S. Visweswaraiya, K.S. Gurumurthy, " A Novel, Dynamic Data Dissemination [D3] Technique for Congestion Avoidance/Control in High Speed Wireless Multimedia Sensor Networks", Fifth 
International Conference on Computational Intelligence, Modelling and Simulation (CIMSim), 2013.

[9] E. Hajian, K. Jamshidi, A. Bohlooli, "Improve energy efficiency routing in WSN by using automata", International Journal of Ad hoc, Sensor \& Ubiquitous Computing (IJASUC) Vol. 1, No.2, 2010.

[10] Li. Zilong, W. Zou, T. Qi, " A cross-layer congestion control strategy in wireless sensor network", in 4th IEEE International Conference on Broadband Network and Multimedia Technology (ICBNMT), 2011.

[11] P. Antoniou, A. Pitsillides, T. Blackwell, A. Engelbrecht, L. Michael," Congestion control in wireless sensor networks based on bird flocking behavior", Computer Networks Volume 57, Issue 5, 7 April 2013, Pages 1167-1191.

[12] Jaiswal, S.; Yadav, A., "Fuzzy based adaptive congestion control in wireless sensor networks," Contemporary Computing (IC3), 2013 Sixth International Conference on , vol., no., pp.433,438, 810 Aug. 2013.

[13] Jutan Wei; Bing Fan; Yi Sun, "A congestion control scheme based on fuzzy logic for wireless sensor networks," Fuzzy Systems and Knowledge Discovery (FSKD), 2012 9th International Conference on , vol., no., pp.501,504, 29-31 May 2012.

[14] H. Cha, K. Kim, S.Yoo, " A node placement algorithm for avoiding congestion regions in wireless sensor networks", in Third International Conference on Ubiquitous and Future Networks (ICUFN), 2011.

[15] J. Mena, V. Kalogeraki, " Dynamic Relay Node Placement in Wireless Sensor Networks" in International Symposium on Applications and the Internet, SAINT ,2008.

\section{Authors}

Ali Dorri received his B.S. degree in computer engineering from Bojnord University, Iran, in 2012, and now is student in M.S in software engineering in Mashhad branch, Islamic Azad University, Mashhad, Iran. His research interests cover Wireless Sensor Networks (WSN), Mobile Ad hoc Network (MANET) and specially Security challenges.

Dr. Seyed Reza Kamel Tabbakh is with the Department of Software Engineering, Faculty of Engineering, Islamic Azad University - Mashhad branch, Mashhad, Iran. He received his $\mathrm{PhD}$ in communication and network engineering from University Putra Malaysia (UPM) in 2011. He received his BSc and MSc in software engineering from Islamic Azad University, Mashhad branch and Islamic Azad University, South Tehran

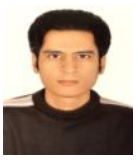
branch, Iran in 1999 and 2001 respectively. His research interests include IPv6 networks, routing and security. During his studies, he has published several papers in International journals and conferences. 